\title{
Physical Properties of Binary Brown Dwarfs
}

\author{
W. Brandner ${ }^{1}$, M. Stumpf ${ }^{1}$, R. Köhler ${ }^{1}$, V. Joergens ${ }^{1}$, F. Hormuth ${ }^{1}$, \\ K. Geißler ${ }^{1}$, B. Goldman ${ }^{1}$, T. Henning ${ }^{1}$, H. Bouy ${ }^{2}$ and E. Martin ${ }^{2}$ \\ ${ }^{1}$ Max Planck Institute for Astronomy \\ ${ }^{2}$ Instituto de Astrofísica de Canarias, Spain
}

\begin{abstract}
Astrometric observations of binary brown dwarfs yield dynamical masses of the components independently of theoretical models. We give an update on our long-term high-resolution spectroscopic and photometric monitoring programme of spatially resolved binary brown dwarfs using ground-based adaptive optics and the Hubble Space Telescope. We present current orbital fits, including refined dynamical mass estimate of the Kelu-1 AB system. The results seem to support the previously reported trend that evolutionary and atmospheric models might underestimate the mass of very-low-mass stars and brown dwarfs.

Alternatively, additional, thus far unresoled system components (i.e. a higher degree of multiplicity) could explain the unexpectedly high dynamical mass estimates. In the case of Kelu-1 $\mathrm{AB}$, we present potential spectroscopic evidence for a third system component. The confirmation of this third system component would resolve the reported discrepancy between evolutionary models and dynamical mass estimates. This might make Kelu-1 AB the first pure brown dwarf triple system discovered so far.
\end{abstract}

